TITRE: ÉTUdE DE MANUSCRITS DE MIGRANTS : LE HORS-NORMES COMME LIEU DE MÉTALANGAGE

Auteur(s): Nathalie Matheu, Doctorante, Praxiling, UMR 5267, UniVersité Paul Valéry Montpellier 3-CNRS

PUBLICATION: ÉCRITS HORS-NORMES

PAGES: $52-64$

Directeurs: Agnès Steuckardt et Karine Collette

ÉdITEUR: LES ÉDITIONS DE L'UNIVERSITÉ DE SHERBROOKE, 2019

ISBN: 978-2-7622-0360-8

URI: HTTP://HDL.HANDLE.NET/11143/15572

DOI: HTTPS://DOI.ORG/10.17118/11143/15572 


\title{
Étude de manuscrits de migrants : le hors- normes comme lieu de métalangage
}

Nathalie Matheu, Doctorante, Praxiling, UMR 5267, Universite̋Paul Valéry Montpellier 3-CNRS

Résumé : En France, le débat autour de la norme a toujours cours. Au niveau linguistique, une normativisation dure a conduit au refus d'installer des normes lexicales, grammaticales, syntaxiques ouvertes. De par son caractère plus stable, l'écriture est devenue un haut lieu de la norme, instituant un renversement de la pensée logocentriste et stigmatisant ceux qui n'ont que peu ou pas de pratiques de l'écrit. Cette attitude est fortement relayée par l'école, qui mêle parfois l'activité métalinguistique nécessaire à l'appréhension de la langue, première ou seconde, et les exigences normatives qui consacrent une langue idéale. Notre recherche se propose ici d'interroger certaines traces graphiques de ce « hors-normes » comme les manifestations d'une activité réflexive du scripteur sur son discours. Le protocole expérimental - des ateliers d'écriture auprès d'adultes et d'enfants migrants apprenants de Français Langue Seconde - a permis de recueillir un corpus de 153 textes. L'analyse de ces textes reconstruit le déroulement du processus scriptural, par le repérage des ratures et ajouts, signes visibles de l'activité métalinguistique et métadiscursive du scripteur. Nous nous intéresserons ici à quelques phénomènes de glose et d'adresse, traces de «hors-normes » dans ce contexte, que nous évaluerons comme indices des interrogations du scripteur sur sa production.

Mots-clés : écriture, atelier d'écriture, migrant, Français Langue Seconde, norme

\begin{abstract}
In France a debate about language teaching/learning standards exists. Strong normalization has prevented the implementation of flexible standards in lexicon, grammar and syntax at a linguistic level. Because written language seems more stable, it has become the standard reference for evaluation, establishing a reversal of logocentric thought and putting those whose don't write at a disadvantage. We find this attitude at school where metalinguistic tasks required to learn mother tongue or foreign language are mixed with standard requirements to speak an ideal language. Our research claims that non-standard written utterances are the evidence of reflexive activity taking place within the writer's discourse. We experimented in writing workshops with adults and migrant school children and we collected 153 texts. The analysis of these texts shows the progress of writing through locating graphic marks: marks that have been erased or added. These marks are the visible signs of the metalinguistic and metadiscoursal activity of the writer. We focus here on some specifications that are clues to the questions of the writer on his/her writing product.
\end{abstract}

Key words: writing, writing workshop, migrant, Second Language French, standard 
Cette recherche s'inscrit dans le cadre plus large d'un doctorat en Sciences du Langage, dont l'objectif est d'analyser, à partir des outils de la génétique textuelle, les dynamiques de l'écriture en Français Langue Seconde, à partir d'un corpus de textes réalisés en atelier d'écriture par des apprenants migrants, adultes et enfants scolarisés. Ces textes manuscrits se présentent au premier abord comme hors des normes morphosyntaxiques attendues par l'école et les institutions d'enseignement (traces de la L1, erreurs, lexèmes en L1...). Nous voudrions postuler que si la langue écrite est le lieu privilégié de la norme, des éléments de ce « hors-normes » vont donner, par l'analyse des pratiques scripturales, des indices à propos de l'activité réflexive du scripteur, sur son discours et sur sa langue d'usage.

Notre méthodologie est ethnographique - nous effectuons des observations participantes sur le terrain et compréhensive, afin de favoriser la réflexivité des apprenants sur leurs propres pratiques d'écriture, notamment dans une optique d'évolution des représentations en jeu et de développement des compétences en langue française. Les ateliers d'écriture sont menés auprès d'adultes migrants en contexte associatif et d'Enfants Nouvellement Arrivés au secondaire. Tous sont en apprentissage du Français Langue Seconde et les ateliers sont proposés en complément de la classe ordinaire. L'atelier d'écriture, parce qu'il se déroule en-dehors des cours explicitement linguistiques, va permettre d'appréhender la langue, et notamment l'écrit, hors de toute appréhension évaluative, afin d'autoriser une manipulation du scriptural différente et stimulante.

\section{La langue écrite comme référence de la norme}

\section{Le renversement de la pensée logocentriste}

L'acte d'écriture reste peu aisé à définir. On trouve de nombreuses acceptions qui ont été déclinées au cours de l'histoire de la littérature, de la linguistique et de la didactique des langues, maternelle ou seconde. L'école n'est donc pas la seule à être en situation de perplexité face à cette notion. Ce flou définitoire tient peut-être en premier lieu à la polysémie même du verbe écrire, polysémie qui est le reflet de la multiplicité des pratiques d'écriture (graphier, transcrire, produire un écrit en tant qu'acte de langage). Au fil du temps ont été valorisées les sociétés ayant une tradition écrite, considérées comme des sociétés qui pensent, qui se pensent car elles s'écrivent et donnent à lire leur histoire. Néanmoins, si l'écriture a un fonctionnement spécifique, rien ne justifie que ce fonctionnement soit supérieur à celui de l'oral. Il est difficile de penser l'écriture sans la confronter à la parole et il nous faut brièvement revenir à leur définition initiale.

Depuis Platon, écriture et parole ont été distinguées. L'éloge de la parole, comme saisissement du logos, ne peut se réaliser qu'à travers la voix. Cette définition est assez antinomique de celle de notre société ou bien de celle de l'école. Pourtant, en dépit des interprétations communément admises, la critique de l'écriture par Platon, comme simple représentation de la voix, n'est pas une condamnation. Platon a luimême beaucoup écrit. La relecture de Luc Brisson du Phèdre dans sa réédition de 2004 apporte un nouvel éclairage sur la dichotomie platonicienne parole/écriture. Par exemple, Platon, par la voix de Socrate, réfute la possibilité pour l'écriture d'appréhender du certain, et pour démonstration, commente l'analogie entre l'écriture et la peinture, qui simule la réalité par l'apparence figée du vivant. Cette analogie est déjà perceptible dans le verbe graphein, qui, en grec ancien, relate à la fois l'acte d'écrire et celui de peindre. Pour Socrate, ce simulacre de réalité touche également les discours, qui perdent toute réalité une fois transcrits. 
Ainsi sont signifiées les limites de l'écriture, dans la mise en place d'une réflexion destinée à expliciter les statuts des discours parlés et écrits. L'écriture est un jeu, dont le philosophe peut user pour se divertir, car il a la conscience des limites de cette pratique. II n'y a donc pas rejet mais « constat lucide » face à un moyen de communication en expansion dans une civilisation de l'écriture « où le problème de la mémorisation des messages à transmettre avait cessé de dominer la vie intellectuelle, et où, par conséquent, l'esprit humain pouvait prendre du recul pour évaluer les avantages et les inconvénients des divers moyens de communication qui s'offraient désormais à lui. »(Brisson, 2004 : 61).

Ainsi, peu à peu, par son caractère plus stable que la langue orale, par la nécessité progressive de sa normativisation, dans une visée de diffusion et d'unification, la langue écrite s'est autonomisée, jusqu'à devenir un système spécifique et fort différent de celui de la langue parlée. Par là même, ses fonctions se sont modifiées. Par son statut linguistique propre, elle possède également des fonctions caractéristiques, et ne relève plus de la simple transcription de la parole. Une sorte de renversement s'est même insidieusement institué : parce qu'elle est un facteur puissant de culture et d'unification, elle est devenue l'image de la langue elle-même, dont le langage oral ne serait qu'une variation, souvent dévalorisée.

La naissance de l'écriture s'accompagne d'une transformation de la parole [...]. La parole d'un peuple d'écriture n'est plus la même. C'est un langage annexe, subordonné à l'écrit. Un langage sans importance, sans pouvoir [...]. Ce qui est jugé important ne passe plus par la parole. La parole n'est plus que conversation, échange anodin. Elle n'est, dans notre société, jamais en rapport avec la vérité, et la preuve : la science ne se sert que de l'écriture. (Pividal in Goody, 1979: 12).

C'est donc ainsi que nos sociétés contemporaines se représentent ou vivent leur relation imaginaire à l'écrit. II s'agit pourtant là d'une représentation réductrice, qui ne donnent pas à voir toute la complexité des enjeux.

\section{L’écriture comme lieu de la norme : la langue écrite comme langage véhiculaire}

Les volontés politiques d'unification des territoires, au cours de l'histoire, se sont affirmées et étendues par le partage d'une langue commune, donc nécessairement normativisées. Il apparaît que d'une manière générale, les formes écrites ont plus de stabilité et donc plus de possibilités de diffusion que les formes orales et « ont été à la base de la constitution des langues nationales des grands États » (Dubois, 2001 : 163). Ainsi, la forme écrite de l'allemand s'est peu à peu unifiée à partir d'un dialecte de moyen allemand, le Schriftdeutsch et est usité dans l'ensemble des régions germanophones: en Allemagne, en Autriche, dans une grande partie de la Suisse et dans de petits secteurs d'autres pays européens. Si l'on observe des différences considérables au niveau des langues parlées, toute personne alphabétisée a donc la possibilité de communiquer par écrit avec tout autre scripteur de ces territoires.

De la même façon, l'arabe dit littéraire, forme écrite de la langue arabe, recouvre des formes de langue très différentes entre elles, nommées arabes dialectaux, dispersées sur de très grands territoires. Les langues idéographiques comme le chinois sont à ce niveau des cas prototypiques, dont la forme écrite utilise un système de signes à même d'écrire des langues entièrement distinctes, avec quelques variations, notamment dans l'usage des accents. 
Si la langue écrite semble donc stable et homogène, il en va tout autrement dans les pratiques scripturales individuelles. Parce que l'écriture est également un moyen de communication différée, elle va permettre des retours en arrière sur le discours, des rectifications, sous la forme de ratures, d'ajouts ou de déplacements, qui vont perturber cette apparente homogénéité.

\section{Dispositif expérimental et analyse du corpus}

\section{Protocole d'expérimentation : des ateliers d'écriture en langue française}

Parce que tout apprentissage met en déséquilibre les précédents savoirs (Auger, 2010) et qu'une certaine insécurité se cristallise particulièrement autour de la langue écrite (Dabène, 1987 ; Barré-de Miniac, 2000), insécurité liée à ses contraintes spécifiques et à ses enjeux socio-professionnels, ce contexte d'expérimentation offre aux scripteurs un cadre rassurant, où leur parole est reconnue et légitimée :

Pour accéder à cet espace de constructions de connaissances ainsi perçu comme dangereux, menaçant, il convient de disposer d'un ailleurs compensatoire, [...] d'un ancrage où notre parole est reconnue valeureuse et d'où elle assigne à autrui des places et des représentations familières. (Coianiz, 2001: 15-16).

Les ateliers d'écriture ont été mis en place sur une durée de deux ans auprès d'adultes migrants et d'une année scolaire pour les enfants en secondaire. Tous les participants étaient alphabétisés en L1 et nous avons recensé, sur la totalité des participants, quinze langues familiales différentes. Les niveaux en production écrite étaient variables : entre A2 et B2 selon les niveaux du CECR. Les textes ont ensuite été transcrits en transcription diplomatique.

Ecrire dans le cadre d'un atelier d'écriture permet de sortir des objectifs strictement fonctionnel et communicationnel, qui limitent l'écriture à la gestion des situations sociales, dans le cadre de relations transactionnelles et interpersonnelles et de la maîtrise du discours théorique, dans le cas des enfants scolarisés. Il s'agit donc d'offrir aux apprenants la possibilité d'une manipulation du scriptural différente de celle qui se pratique généralement à l'école ou en institution,

L'écriture du texte est contrainte à trois niveaux:

- l'espace graphique (une page A4);

- le temps d'écriture (45 minutes);

- la consigne d'écriture, qui va en orienter la narration, par la proposition d'un thème et/ou d'un genre textuel et/ou d'une contrainte formelle.

Néanmoins, la présentation des consignes n'est jamais prescriptive. Il s'agit principalement d'une orientation ayant pour visée de faciliter l'entrée en écriture du scripteur. L'exposition orale de la consigne d'écriture par l'animatrice peut se dérouler selon trois axes possibles suivant la séance:

- présentation du thème, suivie d'une discussion avec les participants, qui permet d'en dégager les possibles orientations. Par exemple, pour l'atelier autour du thème du grenier a été évoqué le lieu situé dans la maison. Le temps de discussion a ensuite permis, en définissant ce qu'était 
le grenier, c'est-à-dire un local destiné au départ au stockage du grain, puis chez les particuliers à remiser les affaires inutiles et les souvenirs, à envisager par extension le grenier comme un lieu de mémoire;

- $\quad$ présentation d'un genre textuel : nous avons travaillé par exemple sur le haïku, qui appelle des contraintes spécifiques, comme la concision, l'ellipse dans la narration;

- $\quad$ présentation d'une contrainte formelle : par exemple la reprise anaphorique. La séance a alors débuté avec le visionnage d’ Un jour», vidéo du poète sonore Charles Pennequin.

Cette mise en scène orale a pour objectif de dépasser le premier stade de lecture de la consigne et en cela peut être apparentée au marmottage qui précède parfois la mise en graphie chez tout scripteur (Penloup, 2007). De la même façon, les textes ne sont pas évalués au regard des normes linguistiques. Les retours sur les textes sont effectués par les autres participants, après le temps de lecture des manuscrits à voix haute par leur scripteur. Ce temps de lecture est fondamental : outre le partage des textes dans le groupe, les textes sont évalués par des lecteurs et non des formateurs. Peuvent ainsi être signalés des difficultés de compréhension qui entraînent le scripteur à s'interroger sur ses partis-pris scripturaux, sur sa syntaxe, son vocabulaire... Peu à peu se met donc en place un cheminement vers des normes objectives, pour faciliter la réception des textes. De plus, parce que les remarques sont toujours bienveillantes et témoignent d'un intérêt collectif, le scripteur se trouve valorisé dans son écriture, ce qui accroîtégalement sa motivation. Ces précautions didactiques tendent à lever - au moins partiellement - la lourdeur des enjeux qui pèsent, pour les migrants, sur l'apprentissage de l'écrit.

Chaque séance débute par l'élaboration d'un inventaire, collectif ou individuel. Par la liste, le savoir est consigné - et ici partagé entre les participants - mais cette consignation n'a pas seulement de fonction taxinomique. J. Goody fait état des listes lexicales, qui semblent s'être développées à titre d'exercices ou de jeux, peut-être pour les scribes. La possible fonction ludique de ces listes de noms ou de mots commençant par la même lettre n'est pas sans évoquer la création d'inventaires pratiquée en atelier d'écriture.

Elles [les listes lexicales] sont le signe d'un travail d'abstraction, de décontextualisation, d'une activité ludique -parfois aussi d'un certain art de s'enfermer dans les concepts. (Goody, 1979: 170).

Ces différents parti-pris didactiques vont libérer le scripteur des exigences normatives strictes et, au-delà d'une remédiation des phénomènes d'insécurité scripturale, l'autoriser à manipuler la langue écrite librement, l'encourager à effectuer des retours sur son texte, des rectifications, qui n'ont pas pour seule visée la correction des erreurs morphosyntaxiques.

\section{L'élaboration du corpus}

Les données recueillies pendant cette recherche se composent:

- d'un corpus audio d'entretiens effectués avec les participants volontaires en début et en fin de cycle de pratique des ateliers;

- d'un corpus audio d'enregistrements des interactions qui se déroulent pendant les pratiques d'écriture ;

- d'un carnet de recherche composé de notes prises pendant le temps d'écriture des participants; 
- d'un corpus de 153 textes manuscrits réalisés par les participants à l'issue de la présentation des consignes d'écriture.

L'ensemble de ces corpus sont transcrits, selon les normes en usage pour les documents audio, et pour les manuscrits en transcription diplomatique.

La multiplication des données a pour visée d'objectiver l'analyse des textes et de mettre en perspective les indices scripturaux avec les intentions du scripteur.

\section{L'écrit comme lieu d'hétérogénéité : des textes « hors-normes »}

\section{Erreur et norme}

Le concept d'erreur s'articule dans le champ de la linguistique autour de la notion d'écart par rapport à une (ou plusieurs) norme(s) ou écart par rapport à la production supposée, dans un même contexte d'énonciation, d'un locuteur natif (Legendre, 2005). Cette définition de l'erreur comme écart par rapport à une référence semble avoir déterminé l'appréhension de la production fautive et sa sanction dans l'enseignement traditionnel :

Les critères d'identification des erreurs, souvent imprécis ou superficiels, sont très divers et parfois - notamment pour le français - très normatifs. Cela s'explique soit par les conditions d'enquête, soit par l'absence d'objectifs précis, soit par la reproduction dans l'enquête de normes pédagogiques restrictives, imputables à l'enseignement ou aux enquêteurs eux-mêmes, comme par exemple le refus de considérer comme correct tout énoncé ou toute forme n'appartenant pas au mythique français standard. (Porquier, 1977 : 24-25).

Il importe donc de s'interroger sur le choix de la norme utilisée comme référence et sur la réalité sociolinguistique de la langue normative (Boyer, 1991). Dans l'Histoire, l'instauration du français comme langue officielle s'est bâtie sur le socle du refus de toute déviance, inter ou intralinguistique. La détermination de ce qui est, ou non, correct, a aussi à voir avec les imaginaires linguistiques et les représentations que l'enseignant véhicule sur sa propre langue.

\section{Un décalage avec les attentes scolaires et institutionnelles}

Les textes de notre corpus se présentent au premier abord comme tout-à-fait éloignés des attentes normatives de l'école. Ils offrent un agencement spatial d'éléments hétérogènes où se mêlent des portions de textes, de longueur variable, plus ou moins raturés, éventuellement ponctués d'ajouts, des zones clairement délimitées par des traits verticaux et/ou horizontaux, parfois des dessins. 


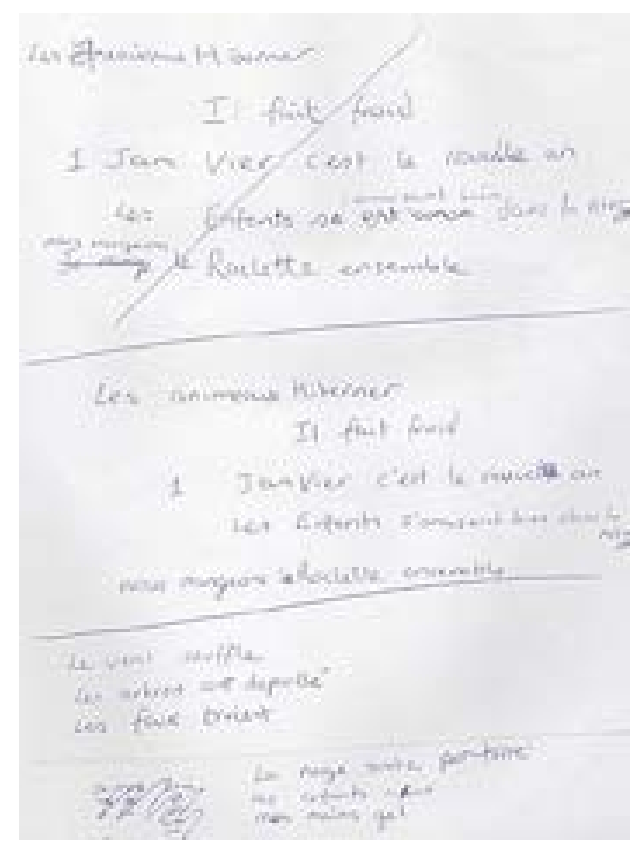

Figure 1. Exemple d'écrit produit par un migrant

Cette composition, en apparence désorganisée, n'est pas sans rappeler les grifouillis (mot-valise composé de gribouillage et de fouillis) chers à Aragon.

Au niveau du texte lui-même, nous pouvons sérier des récurrences, qui sont en décalage normatif avec les attentes institutionnelles. Nous en donnerons ici trois exemples.

\section{Les erreurs morphosyntaxiques}

La langue française a des marques spécifiques, notamment à l'écrit (marques de genre, de nombre, lettres idéographiques, irrégularités morphosyntaxiques) qui peuvent générer des erreurs, en langue première comme en langue seconde. Nous trouvons dans ces textes les erreurs typiques des apprenants, notamment quand ces règles et normes ne sont pas les mêmes dans la langue première des scripteurs:

\section{a. Nous allon chez nos amis \\ b. Je fais couche les enfants}

Ces deux occurrences sont issues de textes d'une apprenante russophone. Nous trouvons des lettres muettes en russe, mais en bien moindre proportion qu'en français. L'oubli des désinences verbales non oralisées est donc fréquent dans notre corpus. Il convient également de préciser que ces erreurs de type a) peuvent également se retrouver dans des corpus d'apprenants francophones, liées à la non oralisation de certaines marques orthographiques. 
Les calques entre $L 1$ et $L 2$ sont également des erreurs très fréquentes, qui apparaissent notamment dans l’ordre erroné des éléments de la phrase:

- une apprenante anglophone

a. Je manque la mer

Il y a inversion du COD et du sujet entre les deux langues.

- une apprenante hispanophone

b. Ma fille n'a voulu pas porte une robe de orange comme soleil.

La place de l'adverbe pas (second terme de la négation) est placé après le verbe au participe passé, comme en espagnol.

\section{Les formulations transcodiques}

La troisième manifestation de ce qui apparaît comme du « hors-normes » relève, suivant la définition de Georges Lüdi, de la formulation transcodique. Si ce concept a été en premier lieu élaboré à partir de corpus d’interactions verbales, il nous semble tout-à-fait transposable dans notre contexte:

La formulation transcodique consiste en un emploi potentiellement conscient, dans un énoncé en langue seconde, d'une séquence perçue par le locuteur non natif comme appartenant à une autre langue (le plus souvent sa langue première), dans le but de surmonter un obstacle communicatif. [...] elle fait partie des stratégies compensatoires interlinguales. (Lüdi, 1993 : 127).

a. Je suis très alèrgique a la picure de mosquito!

Cette apprenante lusophone a utilisé le lexème mosquito en portugais à la place du terme attendu en français, qui lui était inconnu. Afin de ne pas interrompre la rédaction de sa phrase, il y a eu échange entre les lexèmes des deux langues.

Ces trois exemples sont prototypiques des manifestations du « hors-normes » relatives aux textes écrits dans une langue d'apprentissage. Les types d'erreurs sont nombreux et se trouvent à différents niveaux du discours : pragmatique, textuel, phrastique et infraphrastique (Marquilló : 2003). Nous observons également lors de l'analyse longitudinale une diminution, voire une remédiation des erreurs, ce qui implique un cheminement progressif vers des normes objectives, par le développement des compétences en production écrite des participants.

Au-delà des erreurs et des marques transcodiques précédemment décrites, les textes de notre corpus donnent à voir une activité scripturale riche et hétérogène, dans le sens où les ajouts du scripteur vont nous donner des indices sur son appréhension de la langue d’apprentissage et son écrit en train de se faire. 


\section{Le métalangage comme lieu d'hétérogénéité scripturale}

En milieu scolaire ou d'apprentissage, les activités métalangagières, qui permettent aux apprenants d'appréhender le fonctionnement de la langue, sont parfois confondues avec des tâches d'application des règles, pour aller vers la norme scolaire.

L'école est déterminante dans l'institution de l'activité réflexive de type métalinguistique. Cette activité porte à la fois sur le fonctionnement du langage mais aussi sur la valorisation d'un «mieux» dire, conformément à une idéologie de la hiérarchie des formes sur le modèle de la norme prescriptive. (Canut, 2007 : 65).

En France, ces deux activités sont souvent indissociables ou volontairement confondues. Néanmoins, il est important de rappeler que le métalangage est lui-même source d’hétérogénéité. Je renvoie notamment aux distinctions qui ont été o pérées par Jacqueline Authier-Revuz, sur le métalangage portant sur la langue, sur le discours en train de se faire, le discours d'un autre (Authier-Revuz, 1995). Le métalangage est un langage qui vient se superposer, s'interposer dans le flux du discours, qu'il soit oral ou écrit, et par là-même en perturber le cours. Notre corpus est composé de textes au stade de brouillons, c'est-à-dire que restent visibles les traces graphiques qui marquent les retours du scripteur sur son discours. Nous allons voir que des segments apparemment « hors-normes » permettent de mettre au jour une activité métalangagière particulière, notamment par l'utilisation de la glose.

\section{Phénomènes de glose}

Nous voulons ici focaliser sur les phénomènes de glose que nous avons trouvés à plusieurs reprises dans notre corpus. Parce que la glose est une spécification du discours, elle nous semble prototypique de l'alternance des rôles scripteur / lecteur de tout écrivant et témoigne donc d'une évaluation de celui-ci sur son propre texte. Elle témoigne également d'une attention portée au destinataire du texte. La glose n'est pas en soi une manifestation du hors-normes. Elle est répertoriée dans les parties du discours et son fonctionnement a été bien étudié par la communauté scientifique (Steuckardt et Niklas-Salminem, 2003). Néanmoins, les gloses ici rencontrées sont spécifiques à deux niveaux : la variabilité de leurs fonctions ainsi que la multiplicité des destinataires, et en cela se distinguent de la glose plus «normée».

\section{Les doublons lexicaux (L2/L1)}

Une apprenante anglophone a usé régulièrement de ce procédé, qui est somme toute assez fréquent chez les apprenants de langue étrangère. Les traductions en L1 ont en premier lieu une fonction mémorielle et ont donc tendance à s'estomper au fil de l'apprentissage. Néanmoins, par la mise en relation entre les différentes données du corpus (ici le texte et les notes du carnet de recherche), ces doublons ont une fonction complémentaire.
a. Les matins de l'ete (summer mornings)
b. j'aime la vue sur la Pyrânne avec la niege (mountain)
c. Il regarde quelque chose les films d'horror (horror film) et il rire beaucoup
d. I n’aime pas Les guerres, cruauté a les animaux, fois grais [...] fait peur (Bullying) 
Les deux premières occurrences f) et g) sont issues du même texte, l'occurrence h) et l'occurrence i) sont isolées dans des textes distincts. II est à noter que ces lexèmes en L1 sont notés entre parenthèses, c'està-dire hors-texte, ce qui est cohérent avec leur fonction de mémorisation. Ils ne sont alors destinés en premier lieu qu'à la scriptrice, pour d'éventuels retours de lecture ultérieurs. Néanmoins, lors du temps de lecture à voix haute, ces segments lexicaux ont été oralisés avec l'ensemble du texte. Malgré la mise entre parenthèses, ils font donc partie intégrante du texte final car adressés à une destinataire particulière, une autre apprenante anglophone.

Ces ajouts, marqués par les parenthèses, signes graphiques typiques de la glose, ont une valeur explicative. La scriptrice suppose pour la destinataire un risque d'incompréhension des segments précédant sa spécification. L'explicitation sémantique va alors porter:

- $\quad$ sur le groupe nominal (occurrences f) et h) avec une traduction en miroir au niveau de l'agencement grammatical : nom + complément du nom > complément du nom + nom [summer mornings / horror films] ;

- sur un lexème particulier (occurrence g) ), ici la traduction fonctionne comme une synecdoque, en généralisant le particulier : Pyrénées > montagnes [mountain];

- $\quad$ sur une proposition (occurrence i) ). Le procédé explicatif est métonymique : ce qui fait peur > le harcèlement [bullying].

\section{Les spécifications d'ordre interculturel}

\section{Considérons ces deux occurrences j) et $k)$ :}

a. Je me souviens deux guerre, que j'ai n’oublié pas jamais. (c'est en 1994 et en 1999)

b. Quand je dormire dan ma chambre à Mayotte c'êté trés jolie pas ce que il ya de jolie meuble joli décoration et le lit en moustecaire beaucoup des moustiqus a Mayotte et des jolies rideaux

Nous trouvons dans ces deux phrases un élément qui se distingue graphiquement du reste du texte: une mise entre parenthèses en j) et un changement de couleur en k). Chacun de ces éléments ainsi distingués graphiquement va apporter un complément d'informations au syntagme nominal antéposé. La proposition (c'est en 1994 et en 1999) vient dater les deux guerres de Tchétchénie auxquelles la scriptrice fait référence. De la même façon, le syntagme nominal beaucoup des moustiques a Mayotte vient justifier la présence, dans la chambre qui est décrite, d'un lit avec une moustiquaire.

Rappelons que les participants des ateliers sont d'origines diverses et que, même si les parcours migratoires sont étendus, certains pays, leur histoire comme leur climat ou leur faune, sont inconnus à la majorité d'entre eux. Les deux formules appositives ont donc semblées nécessaires aux scriptrices pour expliciter le contexte, historique en j), culturel en k).

\section{Glose d'explicitation grammaticale}

Dans cette dernière occurrence, le syntagme nominal moi et ma sœur est ajouté entre le pronom personnel et le syntagme verbal.

a. moi et ma sœur nous avons peur des voleurs 
Une construction canonique exigerait, outre l'inversion des deux éléments du syntagme nominal, que celui-ci soit placé en début de phrase, comme en m) :

b. Moi et ma sour, nous avons peur des voleurs.

Nous remarquons que le segment moi et ma sœur a été rajouté et n’a donc pas été écrit au fil du texte. Il témoigne donc d'une relecture, puis de la décision d'expliciter le pronom nous. II s'agit également de préciser que cet extrait de corpus est issu d'un inventaire et que nous n'est pas, dans ce cas, une reprise anaphorique.

\section{Conclusion}

Les textes de notre corpus se présentent comme des textes dits « hors-normes »: ils sont en effet en dehors des attentes scolaires et recèlent un certain nombre d'erreurs. Néanmoins, des signes graphiques de cette non adéquation à des normes objectives nous ont donné des renseignements sur les interrogations du scripteur au moment de sa production. L'attention a été portée sur trois phénomènes de glose, qui nous ont semblé éclairer la double posture scripteur/lecteur et l'attention portée à la compréhension du texte par le destinataire.

Le nouvel intérêt porté aux corpus « sortant de l'ordinaire » permet en effet de valoriser des textes jusquelà mis de côté et donnent à voir l'hétérogénéité et la richesse des pratiques scripturales. 


\section{Bibliographie}

Auger Nathalie (2010), Élèves nouvellement arrivés en France, Paris, Éditions des Archives contemporaines.

Authier-Revuz Jacqueline (1995), Ces mots qui ne vont pas de soi. Boucles réflexives et non-coïncidences du dire, Paris, Larousse.

Boyer Henri (1991), Langues en conflit. Etudes sociolinguistiques, Paris, L'Harmattan.

Brisson L. (2004), traduction du Phèdre (6eéd.). Suivi de : La pharmacie de Platon de Jacques Derrida, Paris, Flammarion.

Canut Cécile (2007), Une langue sans qualité, Limoges, Lambert Lucas.

Coïaniz Alain (2001), Apprentissage des langues et subjectivité, Paris, L'Harmattan.

Dabène Michel (1987), L’Adulte et l'écriture, Bruxelles, De Boeck Université.

Dubois Jean et al., (2001), Dictionnaire de linguistique, Paris, Larousse.

Goody Jack (1979), La Raison graphique, Paris, Minuit.

Legendre Renald (2005), Dictionnaire actuel de l'éducation, Montréal, Guérin.

Lüdi G. (1993), « Statuts et fonctions des marques transcodiques en conversation exolingue », G. Hilty (dir.), Actes du XXe Congrès international de linguistique et Philologie romanes. Université de Zürich, 6-11 avril 1992, Tübingen/Basel, Narr, t. III, 123-136.

Marquilló Larruy Martine (2003), L'Interprétation de l'erreur, Paris, Éditions CLE International, coll. « Didactique des Langues Étrangères ».

Penloup Marie-Claude (2007), «Place et rôle de l'oral dans les ateliers d'écriture », J. Foucault (dir.), Ateliers d'écriture. Journée d'étude de Lomé (Togo), Paris, L'Harmattan, coll. « Lignes d'écriture », 53-64.

Porquier Rémy (1977), "L'analyse des erreurs : problèmes et perspectives », Études de Linguistique Appliquée, n’25, 23-43.

Steuckardt Agnès et Niklas-Salminen Aïno (dir) (2003), Le Mot et sa glose, Aix-en-Provence, Publications de l'Université de Provence. 